\title{
Celiac Disease Hiding Behind Cerebral White Matter Lesions: A Case Report
}

\author{
Mohammed Elhassan $^{1, *}$, Guha Venkatraman ${ }^{2}$ \\ ${ }^{1}$ Department of Medicine, University of California in San Francisco (UCSF)/Fresno, Fresno, CA, USA \\ ${ }^{2}$ Department of Medicine, St. Barnabas Medical Center, Livingston, NJ, USA \\ *Corresponding author: melhassan@fresno.ucsf.edu
}

\begin{abstract}
Celiac disease, or celiac enteropathy, is a relatively common autoimmune disease with myriad of presentations, including intestinal and extra-intestinal manifestations. It is also a less common cause of cerebral white matter lesions in the absence of other etiologies. If such patients are symptomatic, diagnosis can be challenging, especially when gastrointestinal symptoms are absent. The differential diagnosis in such cases is wide and high index of suspicion is needed to make the diagnosis. We presented a case of celiac disease who presented with neurological symptoms and white matter lesions in brain magnetic resonance imaging without gastrointestinal symptoms.
\end{abstract}

\section{Keywords: celiac disease, leukoencephalopathies}

Cite This Article: Mohammed Elhassan, and Guha Venkatraman, "Celiac Disease Hiding Behind Cerebral White Matter Lesions: A Case Report." International Journal of Celiac Disease, vol. 5, no. 2 (2017): 81-82. doi: 10.12691/ijcd-5-2-3.

\section{Background}

Celiac disease (CD) is common (almost $1 \%$ of the Western population [1]) and has many extra-gastrointestinal manifestations [2]. Some neurological complications such as ataxia, peripheral neuropathy, and epilepsy are wellrecognized [3]. However, neurological symptoms with white matter lesions (WMLs) in brain imaging without specific underlying etiology is a less recognized variant $[4,5]$. Here we describe a patient who suffered from unexplained neurological symptoms without accompanying gastrointestinal manifestations and with brain MRI showing WMLs who was found, after extensive work up, to have CD.

\section{Case Report}

A 58-year-old woman with a history of depression and aortic regurgitation was referred for neurological evaluation as she was suffering from intermittent headaches and impaired memory for three years and episodic left arm and leg weakness for one year. She attributed her symptoms to an injury she endured three years ago, when she fell and hit the back of her head. She denied diarrhea or weight loss and did not report history of alcohol or illicit drug sue. Family history was not significant for gastrointestinal or neurological disease. On neurological exam, the patient was fully oriented, cooperative, and had no speech or short term memory deficits. Cranial nerves, motor function and coordination were intact. The sensory exam was remarkable for a mild decrease in vibration sense in the right foot. The rest of her examination was unremarkable. Brain magnetic resonance imaging (MRI) showed nonspecific WMLs scattered in the left frontal and parietal lobes without enhancement. Laboratory studies were unyielding, including: complete blood count, complete metabolic panel, thyroid function tests, serum iron, vitamin B12, vitamin E, and heavy metals. Evaluation for Human Immunodeficiency Virus, Human T-Lymphotropic Virus 1 and 2, Lyme disease, Ehrlichiosis, Babesiosis, and anaplasmosis were unremarkable. Additionally, autoimmune antibodies were not present and examination of cerebrospinal fluid including assessment for oligoclonal bands was unremarkable. Magnetic resonance angiography (MRA) of the head and neck did not show stenotic or vasculitic changes and a 72-hour ambulatory electroencephalogram did not reveal seizure activity. In an attempt to find other rare causes of her clinical picture, celiac disease work up was requested. Anti-tissue transglutaminase IgG, anti-gliadin IgA and anti-gliadin IgG came back positive at $49 \mathrm{U} / \mathrm{ml}($ normal $<5$ ), 27 (normal $<11$ ), and 25 (normal $<11$ ), respectively. Subsequently, the patient underwent duodenal biopsy and CD was confirmed with Marsh 3c histology. She was referred to a center specialized in treating patients with celiac disease and was started with intravenous immunoglobulins in addition to the gluten-free diet as decided by the specialist, with subsequent improvement in her symptoms and decrease in her antibody titers.

\section{Discussion}

This case supports the notion that evaluation for $\mathrm{CD}$ is reasonable part of the work-up of patients with neurological symptoms when etiology is unknown, particularly with the presence of WMLs, even in the absence of enteropathy. 
Such MRI lesions, which are non-specific, vary from numerous foci to wide spread confluent pattern in both hemispheres. The WMLs in CD may be due to vasculitis or demyelination but they are generally non-specific and are also seen in patients with cardiovascular risk factors. Headache seems to be the dominant symptom in most similar cases reported. Most of these patients, however, were already diagnosed with $\mathrm{CD}$, unlike our patient. The absence of enteropathy, family history of CD or other autoimmune disease can make it hard to seek the diagnosis of $\mathrm{CD}$ and high index of suspicion is needed when other more common disease entities like multiple sclerosis are not found. Even when serological tests for $\mathrm{CD}$ are positive, diagnosis is such cases needs to be confirmed with small bowel biopsy, since treatment implies major lifelong changes in dietary lifestyle of patients.

\section{References}

[1] Dube C, Rostom A, Sy R, et al. The prevalence of celiac disease in average-risk and at-risk Western European populations: A systematic review. Gastroenterology. 2005;128:S57-67.

[2] Freeman HJ. Clinical spectrum of biopsy-defined celiac disease in the elderly. Can J Gastroenterology. 1995;9:42-6.

[3] Luostarinen L, Pirttila T, Collin P. Celiac disease presenting with neurological disorders. Eur Neurol. 1999;42:132-5.

[4] Hadjivassiliou M, Grunewald RA, Lawden N, el al. headache and CNS white matter abnormalities associated with gluten sensitivity. Neurology 2001; 56:385-8.

[5] Hadjivassiliou M, Currie S, et al. Should we be nervous about celiac disease? Brain abnormalities in patients with celiac disease referred for neurological opinion. Journal of Neurology, Neurosurgery, and Psychiatry 2002 Dec; 83(12): 1216-21. 